\title{
Kirkir Warmi: identidad y rol de la mujer aymara en el desarrollo musical del norte chileno ${ }^{1}$
}

\author{
Kirkir Warmi: Identity and Role of Aymara Women in the \\ Musical Development of Northern Chile
}

\author{
por \\ Manuel Mamani M. \\ Universidad de Tarapacá \\ mmmartes@hotmail.com
}

La concepción básica de dualidad es uno de los principios de la cultura andina en la que está inserta la mujer aymara. Dualidad es una estructura basada sobre un conjunto de pautas y valores que persisten en la actualidad dentro de la sociedad andina. Según esa pauta, cada componente de la sociedad posee una clara conciencia de su identidad, de su singularidad, de sus posibilidades y los roles de su competencia en la sociedad.

En esta interacción dual y colectiva, la mujer aymara cumple funciones que se orientan hacia el sentido dual, denominado panipacha. Esta interacción implica el sentido de solidaridad, reciprocidad e igualdad de condiciones entre el hombre y la mujer, de acuerdo a una escala de valores individuales o colectivos de la cultura andina. Cada persona está regulada por estas pautas dadas por la misma sociedad, por lo que la teoría de reciprocidad y complementariedad forma parte del sistema de distribución y equilibrio aymara.

Palabras clave: mujer, dualidad, música, valores e identidad.

Aymara women are inserted in the basic conception of duality as one of the basic principles of the Andean culture. Duality is a structure based upon a group of rules and values which are still valid in the Andean society. According to these rules, each member of the society is clearly aware of his/her identity, of his/her singularity, of his/her possibilities and of his/her roles that he she is expected to play within the Aymara society.

In this dual and collective interaction, Aymara women fulfill functions oriented to this dual conception, denominated panipacha. This interaction is congruous with the sense of solidarity, reciprocity and the equality of conditions of men and women, following a scale of individual and collective values of the Andean culture. Each person is regulated by these rules provided by the same society. Therefore the theory of reciprocity and of mutual complement is part of the Aymara system of distribution and balance.

Keywords women, duality, music, values, identity.

\section{INTRODUCGIÓN}

Desde una perspectiva sociocultural andina, la concepción básica de dualidad, en la que está inserta la mujer aymara, es la estructura basada sobre un conjunto de

${ }^{1}$ Este artículo es una versión de la ponencia presentada al IV Congreso Nacional de Musicología, Santiago de Chile. 
pautas y valores aymaras que persiste en esta era contemporánea. Según esa visión, cada componente de la sociedad andina posee una clara conciencia de su identidad, de su singularidad, de sus posibilidades, y los roles de su competencia en la sociedad.

En esta interacción dual y colectiva, la mujer andina cumple funciones que se orientan hacia el sentido de dualidad denominado panipacha. Esta interacción implica el sentido de solidaridad, reciprocidad e igualdad de condiciones entre el hombre y la mujer. De este modo, de acuerdo a una escala de valores de la cultura andina, cada persona está regulada por pautas dadas por la sociedad, por lo que la reciprocidad y complementariedad forman parte natural en la estructura y se hacen efectivas en la distribución y equilibrio en las tareas del mundo aymara.

En lo metodológico, para el trabajo se plantea una hipótesis que permita comprobar y esclarecer la efectividad de las habilidades de la mujer en el campo musical y comprender su sentido semántico, enfatizando los aspectos musicales dentro de una acción dual y colectiva.

Los datos e informaciones fueron recopilados en diferentes contextos sociales y religiosos, en los que se obtuvieron valiosas informaciones mediante grabaciones y entrevistas a las familias, además de la consulta bibliográfica sobre la temática.

El marco geográfico de este estudio corresponde a los poblados de la precordillera y la franja altiplánica de la región de Arica-Parinacota y de Tarapacá.

A través de este trabajo se da cuenta del aporte musical y de la participación de la mujer aymara en los contextos socioculturales y la religiosidad andina, en los que la música juega un papel primordial. Asimismo, se explora la posición e identidad de la mujer relacionada con la música, junto con el rol que ejerce en diferentes ámbitos de la vida andina del norte chileno.

\section{ANTECEDENTES PREVIOS}

La relación de la mujer con la música descansa en la conciencia individual y social andina, modelada y sistematizada por la misma sociedad. En efecto, la mujer aymara no sólo vive compartiendo hábitos, ideas, creencias en la comunidad, sino que ella misma interactúa dentro del quehacer aymara, respondiéndose mutuamente las acciones con la comunidad y su contraparte, con expectativas personales y colectivas. Surge así la relación y equilibrio en la complementariedad andina ${ }^{2}$.

Cuando hablamos de complementariedad, colocamos el acento en el proceso de dualidad: Panipacha; y cuando hablamos de relación, fijamos la atención en la forma que interactúa en la equidad y complementariedad, con los deberes y los derechos de la mujer en un contexto social y económico. Así, el accionar de la mujer se observa y se aprecia por su papel interactivo, en que ambos componentes se relacionan permanente y solidariamente sobre el principio básico de dualidad e identidad ${ }^{3}$.

${ }^{2}$ Espinoza 1990: 131.

${ }^{3}$ Mamani 1989: 17. 
El concepto de desarrollo musical, en el que la mujer aymara tiene injerencia, hace un breve recuento respecto de su aporte en el proceso sincrónico de la música. Hay evidencia de que antes del período Tawantinsuyo la mujer andina había alcanzado un alto prestigio en el campo del arte y la música, estructurado y sistematizado por las sociedades antiguas. Por otra parte, las habilidades innatas de la mujer para la música y otros campos del saber andino también han sido desarrolladas por ella en el campo de la textilería aymara,

\subsection{La música en el Imperio Inca}

El aporte de la mujer aymara en la música y el baile estaba muy extendido por toda el área andina, y muy arraigado a las actividades tradicionales de la zona andina en general. Por ejemplo, en la corte incaica, el sapanica y la coya tenían sus grupos musicales propios. Igualmente, en los acllahuasis (casa de las doncellas), un grupo de personas se dedicaban a las taqui acllas, que practicaban la música y la danza. Las acllas danzaban y cantaban durante las fiestas solemnes con la presencia de altas personalidades andinas. En todas las capas sociales a la música y al baile se les daba la mayor importancia, en particular a la música, por lo que era considerada como un elemento primordial en el Imperio Inca ${ }^{4}$.

\subsection{Significado de cantos y bailes}

El canto y el baile en su conjunto recibían el nombre genérico de taki en quechua y kirki, en aymara, términos que significan acción de cantar y danzar, conjugando ritmos, textos y la plasticidad corporal de las ejecutantes de bailes. Cada actividad cultural era comunicada con un determinado tipo de música y baile, en cuyas figuras y gestos se simbolizaban o reproducían las escenas más importantes en el quehacer musical andino. En otros ámbitos, por ejemplo, en la agricultura (siembra, cosecha y limpia de canales), en la ganadería (ritos de reproducción de animales), en lo social (matrimonios, nacimientos, ritos de iniciación y finalización de acciones), era importante la presencia musical. Mediante cantos y bailes se escenificaban los hechos históricos, míticos, religiosos y legendarios, reviviéndolos ${ }^{5}$.

\subsection{Indumentaria y música}

La música, el baile y el teatro estaban arraigados en lo religioso pagano, por lo que la gente andina se daba cierta 'libertad' en las actividades religiosas sociales, vistiéndose con sus mejores galas, tocados y trajes bellamente adornados, junto a los hombres que también presentaban sus mejores vestuarios. En los documentos musicales más antiguos se menciona el huayno, que era la expresión más común en el incario, como los awatiri (pastores), los satiris (agricultores) y los pasisris (cargos rituales) en los carnavales (alegría y galanteo). Todo tipo de canto estaba relacionado con los ritos a los entes tutelares, cuyos festejos se realizaban para

${ }^{4}$ Espinoza 1990: 401.

${ }^{5}$ Espinoza 1990: 40. 
provocar la fertilidad de la tierra y el ganado, que revestía un carácter orgiástico, como es mencionado en el documento Huarochirí ${ }^{6}$. El baile y la música de casayaco era dedicado en homenaje a la deidad Killa (luna en quechua), considerada como protectora de salud de la mujer. En el área andina central era muy frecuente escuchar la música y ver la danza en los recintos oficiales. En la actualidad continúan estas prácticas, con otra modalidad, a modo de ejemplo, en la casa de los pasantes del rito, en las sedes sociales, con las ideas y sistemas antiguos de los pueblos andinos de esta zona.

\subsection{La música y género}

El wayñu en el tiempo incaico era entonado por las damas y doncellas que percutían la tinya (membranófono), mientras los varones respondían ejecutando flautas de hueso de aves. En el Contisuyo existía una variedad de wayñu en que se danzaban e interpretaban canciones rituales. También conocían la takicachigua, danza erótica, pero elegante, que gustaba a toda la sociedad o ayllu; el llamaya, cantada por las pastoras de animales domésticos, como la llama y alpaca; el quisquina collina, muy preferido por las collas; así como otras para atraer abundantes lluvias, por ende aguas, y para ahuyentar los malos augurios ${ }^{7}$.

\subsection{La música y el ayni}

La música tenía mucha importancia en las actividades laborales colectivas, ya que jugaba un importante rol en el ayni, reciprocidad andina. El canto musical era la alianza para llenar el espíritu y, a la vez, llenar de alegría a los participantes y trabajadores de la tierra, generando ambientes propicios en las tareas colectivas. Era costumbre, de parte de quienes tenían a su cargo la organización de ritos y festejos, esmerarse en obtener la presencia de músicos y cantores por el sistema de ayni. Para las ceremonias de mayor envergadura se preparaban instrumentos musicales con cañas de bambú, y también se fabricaban trompetas de oro, de plata y de cobre, para acompañar los bailes rituales importantes y para convocar las fiestas en homenaje a las deidades ancestrales de la naturaleza.

La sociedad incaica de alta jerarquía tenía también sus propios bailes y música, para ser ejecutada durante las solemnes festividades de Intiraymi, junto a otras. Los incas tenían, incluso, casas para almacenar o guardar los instrumentos musicales, pues para ellos la música tenía mucho valor. Las expresiones preferidas eran el huayno, ejecutado por parejas, y la cachua, ejecutada en coro. En las presentaciones se encontraban las aranyani, danza de enmascarados, y la puruaya, danza para la procesión funeraria, que conformaba un marco ceremonial típico para la representación incaica ${ }^{8}$.

\footnotetext{
${ }^{6}$ Documento manuscrito en quechua sobre los ritos y tradiciones andinos de comienzos del siglo XVII, según Francisco de Avila, citado por Espinoza; 1990: 401.

${ }^{7}$ Espinoza 1990: 402.

${ }^{8}$ Espinoza 1990: 405.
} 


\section{MUJER ANDINA EN EL TIEMPO COLONIAL}

Según estudios antropológicos de la conquista y de la colonia, los europeos implantaron la función evangelizadora dentro de un proceso impositivo marcado por la crueldad. La invocación de idolatrías, con la mujer como su protagonista principal de las prácticas mágico-religiosas, fue considerada funesta por la colonia europea. En el devenir del tiempo, la población andina descubrió canales o estrategias adecuadas para mantener su ideología religiosa, su música, sus creencias y su lengua, al igual que otras sociedades nativas de América.

A pesar de todos estos acontecimientos negativos, los andinos mantuvieron sus rasgos fundamentales, tales como lengua, tradiciones y creencias. Por otra parte, el aislamiento de la gente andina en zonas geográficas abruptas (precordillera y altiplano) fue favorable y estratégico para la conservación de la sabiduría aymara, debido a que el acceso a estos lugares era restringido por la baja temperatura de la zona. Desde entonces hasta el presente, las prácticas rituales, la lengua y la música han venido persistiendo en aquellas regiones. Este legado de sabiduría fue mantenido y practicado por andinos contemporáneos descendientes y herederos de esta rica cultura aymara. Gracias a las mujeres antiguas, que propiciaron su continuidad y práctica, es que aún podemos apreciar estas modalidades de ritos y festejos tradicionales ${ }^{9}$.

\section{IDENTIDAD E IGUALDAD DE LA MUJER}

El mecanismo de la estructura social aymara se desarrolla con sentido de solidaridad e igualdad de condiciones entre la mujer y el hombre. Esta condición básica sustenta el principio de igualdad y equidad de la mujer, no habiendo discriminación en el modelo social andino. La estabilidad conyugal de la mujer no sólo se apoya sobre las necesidades de bienes y servicios, la división de tareas, sino que se fundamenta además sobre la base de la identidad individual y conyugal, con el reconocimiento de la sociedad andina como un cuerpo dual. Este cuerpo dual en la música aymara tiene como finalidad el desarrollo de sus potencialidades para el arte en general y para la música en particular. En efecto, la realidad existencial e histórica de la mujer es la capacidad de ejercer individual, dual y colectivamente dentro de una estructura social, en concordancia con los modelos aymara, con identidad sociocultural, la que constituye el fundamento básico de la vida andina ${ }^{10}$. Por modelo y principio andino entendemos la igualdad de condiciones entre la mujer y el hombre, en que la jerarquía y posición permanecen en ambos componentes: Panipacha. Cada integrante de la sociedad aymara posee una clara conciencia de su identidad, de sus posibilidades y los roles de su competencia en la sociedad ${ }^{11}$.

Desde esta perspectiva, la idea básica de este enfoque es la concepción de dualidad que caracteriza a la mujer aymara, cuya función está basada sobre un conjunto de pautas y valores que aún persiste en la cultura andina.

${ }^{9}$ Mamani 1999: 309.

${ }^{10}$ Harris 1978: 25.

${ }^{11}$ Mamani 1999: 308. 


\section{DIVISIÓN DE TAREAS Y LA MÚSICA}

En los tiempos antiguos, las primeras divisiones de trabajo entre la mujer y el hombre se atribuyen a la época de los cazadores de animales y recolectores de frutos. Los hombres se dedicaban más a la caza de animales silvestres de la época para las necesidades básicas de alimentación, y las mujeres se dedicaban de preferencia a la recolección de frutos, cuidado de animales domésticos, perfección de los tejidos, etc. La conformación física de los hombres le permitía perseguir con mayor velocidad a la presa que las mujeres, lo que era decisivo para asegurarse de los alimentos. En cambio, las mujeres no podían alejarse mucho del asentamiento por posible embarazo, lactancia, cuidado de los hijos, etc. En este período la mujer era favorecida en el conocimiento y la práctica de utilidades herbáticas y el uso de la música en la medicina y en otros campos del saber ${ }^{12}$.

En diversos estudios sobre la división de tareas se puede apreciar cierta concordancia entre los autores de sociología y de antropología cultural. La mayoría de ellos tienden a ubicar o relegar a la mujer en categorías secundarias o inferiores, privilegiando al hombre en primer plano, en desmedro de la mujer. En cambio, en el modelo aymara, la mujer tiene la misma posición que el hombre y ostenta la misma jerarquía. Entonces, ambos componentes están situados en el mismo 'nivel', donde cada uno ejerce su rol correspondiente ${ }^{13}$.

\section{INTERACCIÓN Y DUALIDAD EN TAREAS ANDINAS}

En la interacción social y laboral existen pautas que regulan la dualidad, dándole sentido a la acepción de panipacha (ambas) en concordancia con los valores socioculturales y económicos aymara. La interacción social implica el sentido de solidaridad, reciprocidad e igualdad de condiciones de la mujer respecto al hombre. Cada persona está regulada por pautas dadas por la familia y la sociedad a la que pertenece. La identidad y dualidad forman parte de los valores y se hacen efectivos en la distribución y equilibrio de las tareas, que se van plasmando en forma natural en las labores y la dualidad de la mujer ${ }^{14}$. Tomando la noción de complementariedad, el Dr. Tristan Platt plantea dos tipos de relaciones: la simetría y la asimetría. El primer tipo se basa en la búsqueda de la semejanza, el segundo en la diferencia. Ambos ofrecen modelos contrastados para expresar las relaciones entre oposiciones con miras a la unidad ${ }^{15}$.

\subsection{Acciones de dualidad}

En el contexto de complementariedad se pueden observar las acciones duales entre el hombre y la mujer. Estas tareas complementarias se aprecian en diferentes ámbitos sociales, culturales, laborales y religiosos del área andina de la zona norte chilena ${ }^{16}$.

\footnotetext{
${ }^{12}$ Gavilán 1996: 62; Arnold 1994: 105.

${ }^{13}$ Mamani 1999: 310.

14Arnold 1994; Mamani 1989: 122.

${ }^{15}$ Isbell 1987; Platt 1988.

${ }^{16}$ Mamani 1999: 309.
} 


\section{a) Tareas agrícolas de Pachallampi}

En las actividades agrícolas llamadas Pachallampi se realizan acciones de dualidad en la siembra de papas en la precordillera de esta región. Este trabajo dual consiste en que mientras el varón abre la tierra con la herramienta llamada chonta o liwjana, la dama coloca las semillas de papas para su producción.

b) Marcación de llamas

Se realizan las acciones de dualidad mediante las tareas de marcación de animales domésticos. Mientras el varón sujeta por las orejas a la llama o alpaca, la mujer coloca el adorno llamado chimpu y el zarcillo.

c) Tareas de interacción musical

En las ejecuciones musicales es costumbre realizar acciones de dualidad. Mientras la mujer canta la canción, el hombre ejecuta instrumentos musicales de cuerda, viento o percusión. A modo de ejemplo, en el rito de la Cruz de Mayo el guitarrero entona la estrofa de la canción Cuculí y las mujeres repiten la melodía y estrofa de la canción.

\section{d) Tareas en techumbre de casa}

En el techumbre de casa, uta wayllaña, las acciones son compartidas entre la mujer y el hombre. Mientras las mujeres preparan la waylla, porción de paja brava untada en barro, los varones techan sobre los tijerales en lo alto de la casa en construcción.

\section{e) Investidura de cargos}

En las fiestas patronales es norma o costumbre, en algunos sectores del área andina, efectuar acciones de dualidad de ambos componentes del cargo ritual. La mujer, mediante el baile, coloca el poncho de vicuña al alférez varón y el hombre coloca la manta al alférez dama, lo que los convierte en personajes principales del desarrollo de la fiesta patronal.

Estas acciones de dualidad se realizan mediante textos alusivos y música propia de cada acción o tarea. Algunas acciones se realizan con bailes, como es el caso de la investidura de personajes rituales.

\section{ROL E IDENTIDAD DE LA MUJER AYMARA}

La música y la danza tienen importante significado para la sociedad aymara, por su función de comunicación entre la comunidad y los poderes sobrenaturales del espacio. Así, a través del texto ritual, es posible dialogar mediante la invocación religiosa el bienestar de la comunidad en general, y particularmente de la mujer aymara. La música, como una fuerza dinámica, refuerza los sentimientos propios de la gente andina, enviando mensajes que intentan encontrar el equilibrio de la familia y la comunidad, entre ésta y las deidades, enlazando el sentido de espiri- 
tualidad y simbolismo recurrente en el proceso de la vida andina. La música, como un elemento unificador y comunicador, aporta su accionar y dinamismo ideológico, que expresa mensajes colectivos e individuales andinos ${ }^{17}$.

\subsection{Rol de la mujer en la música}

Dentro de la complementariedad, distribución y equilibrio de las actividades tradicionales, la música constituye un valor insustituible, tanto en los tiempos antiguos como contemporáneos. Por lo mismo, la mujer aymara continúa jugando un importante papel en la estructura social y cultural andina. El sistema de ritos y creencias provee valores sociales que refuerzan y actualizan el pensamiento y espiritualidad en el quehacer aymara. Por lo mismo, la música es uno de los elementos de enlace y comunicación más efectivos entre las comunidades y las fuerzas sobrenaturales. La música, a través de ritos y ceremonias, marca y simboliza las diferentes etapas del ser humano, con un especial significado y relevancia dentro de la estructura social y cultural andina.

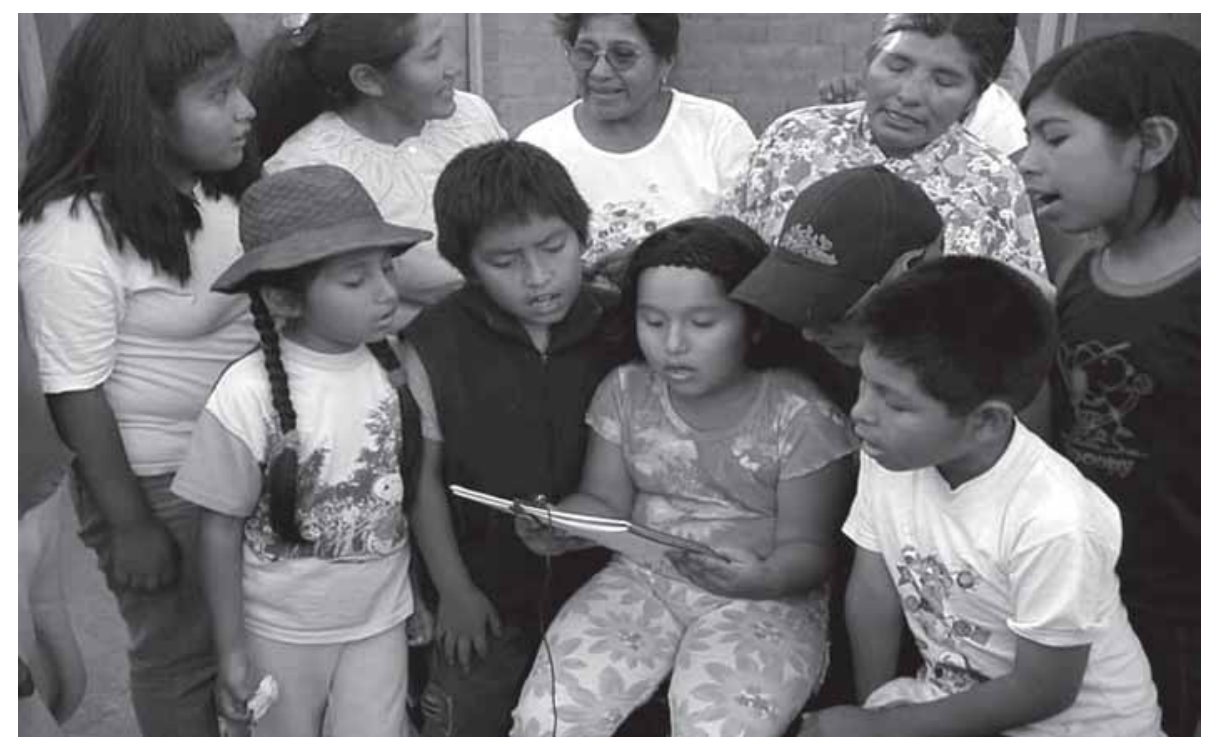

Las niñas y sus madres, con algunos niños, practicando una canción andina con texto aymara. Foto Manuel Mamani.

\subsection{Transición de la mujer y la música}

En el campo de las expresiones musicales, las ejecuciones son compartidas entre la mujer y el hombre. Por ejemplo, las mujeres tienen mayor injerencia en el canto, mientras que los varones tienen mayor injerencia en la ejecución de instrumentos musicales. En estas acciones se observa la complementariedad de ambos compo-

\footnotetext{
${ }^{17}$ Harris 1978: 32.
} 
nentes, si bien los varones también tienen aptitudes para el canto, pero en menor proporción. En estas tareas complementarias, mientras las mujeres cantan la melodía, los hombres ejecutan instrumentos musicales de cuerda, viento o percusión ${ }^{18}$.

Para la mujer aymara, la música es particularmente importante en el proceso de la vida. También está presente en sus actividades cotidianas y eventos tradicionales, al igual que en las tareas pastoriles, agrícolas y textiles, tanto grupales como individuales. La música tiene una real importancia en las diferentes etapas de transición de la mujer y está presente desde la niñez hasta la ancianidad. Esta información sonora es adquirida en su medio familiar y comunal ${ }^{19}$.

\section{a) Etapa de niñez}

En esta etapa la música es practicada en sus juegos infantiles, imitando algunas realizaciones tradicionales de su medio familiar, tales como festividades, costumbres y rituales, que efectúan las mujeres aymara.

\section{b) Etapa adolescente}

El uso de la música tiene importancia en la vida adolescente a través de cantos y bailes propios del género femenino. El niño y la niña procuran buscar la alianza de la música, tanto en las tareas propias de su edad como en las actividades de labranza, de pastoreo de animales, tareas agrícolas y en los juegos grupales con sus pares, en los que la música es de uso primordial.

\section{c) Etapa de juventud}

La música significa para la juventud aymara de ambos sexos un elemento de gran valor durante su vida juvenil. La ejecución de cantos en las jóvenes y la ejecución de instrumentos musicales de los jóvenes se realiza con las tareas propias de la juventud, tanto en los pastoreos de animales, tareas agrícolas, en los viajes y caminatas, etc. La música se hace más relevante para la juventud en las fiestas de carnaval, en otros eventos tradicionales y en galanteos amorosos, así como en las fiestas patronales, en las tareas textiles y en las ceremonias sociales.

\section{d) Etapa adulta}

En la etapa de adultez la música cobra mayor profundidad en las acciones tradicionales insertas en la estructura aymara. La práctica de la música se realiza en los cargos tradicionales, tales como la mayordomía, alferazgos y cabecillas. También en el marco de las ceremonias, ritos, festejos de carnaval y de santos patronos, en las tareas textiles, pastoriles, agrícolas, tareas hogareñas, etc. Es la etapa que la persona alcanza la madurez en el cumplimiento de sus deberes y normas dentro de la estructura social y económica andina.

\footnotetext{
${ }^{18}$ Harris 1980: 73; Mamani 1989: 87.

${ }^{19}$ Mamani 1989: 31.
} 
e) Etapa de ancianidad

La etapa de ancianidad, por su conocimiento profundo, experiencia y sabiduría heredada de sus antepasados, convierte a la mujer en celosa guardiana de las costumbres y tradiciones del mundo andino. En esta etapa la mujer aymara es consejera y guía en las normativas del quehacer aymara, así como en las ritualidades y costumbres de esa sociedad.

\section{APORTE MUSICAL DE LA MUJER}

Las cualidades innatas para el canto y el baile colocan a la mujer aymara en el sitial de preferencia de las reuniones sociales, junto a los festejos religiosos y rituales, que traen consigo un bagaje de conocimientos y experiencias, como herencia de sus antepasados o por su cualidad innata para la música. Este aporte musical de la mujer aymara viene desde tiempos remotos, dentro del sistema social, cultural y económico andino. Por lo mismo, la mujer ha jugado un rol relevante en el desarrollo de la sociedad andina, lo que puede evidenciarse con mayor claridad en las diferentes etapas y actividades culturales y religiosas de la zona norte de nuestro país.

\subsection{Identidad de la mujer}

La identidad musical de la mujer aymara es caracterizada por el timbre de su voz aguda, la que sobresale en una ejecución grupal musicalmente compartida. En este accionar la voz de la mujer imprime e identifica diferentes ámbitos del quehacer andino.

\section{a) Cantos de la Cruz de Mayo}

En el desarrollo de la ritualidad de la Cruz de Mayo, en el accionar del canto y baile (en ronda), el guitarrero entona o canta la frase de la canción de Kukulíy los asistentes, en rueda, repiten dichas estrofas, en donde se destaca la voz aguda de la mujer aymara (ver ejemplo $\mathrm{N}^{\circ} 1$ ).

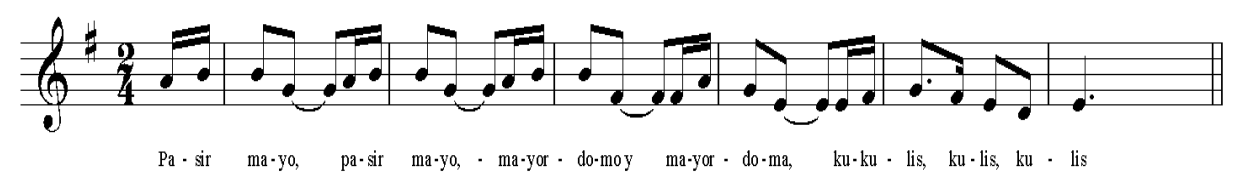

Ej. N 1. Kukuli: Canto Cruz de Mayo. Recopilación y transcripción de Manuel Mamani. 


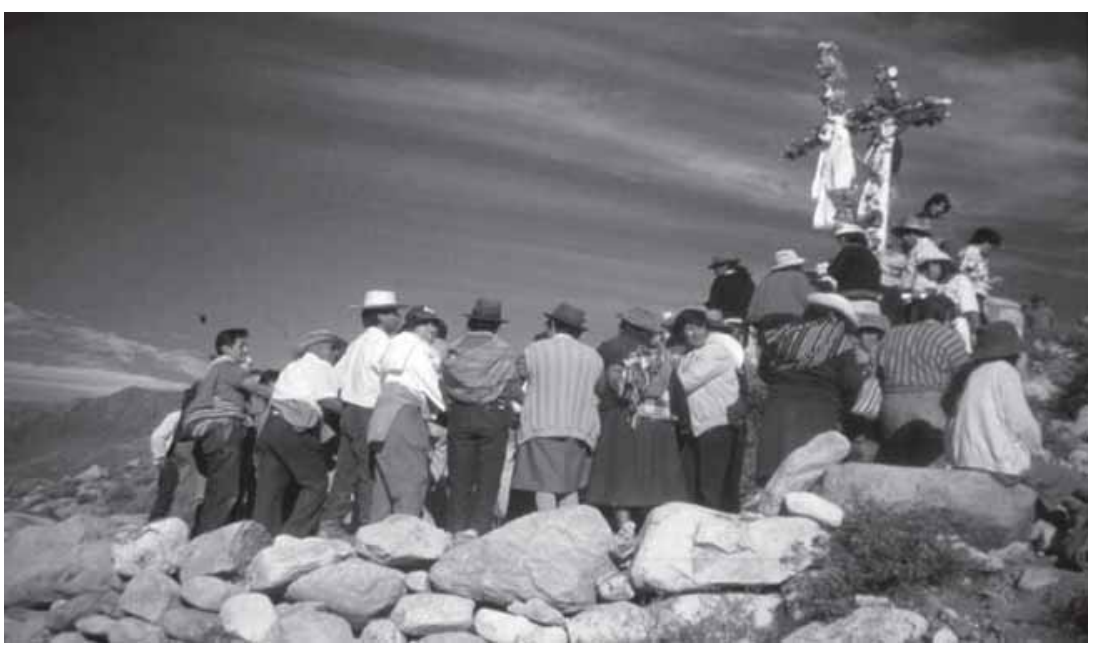

Cruz de Mayo en el Cerro Calvario de Socoroma. Foto Manuel Mamani.

b) Marcación y floreo de ganado

En el desarrollo de la ritualidad de marcación y floreo de animales domésticos, tales como la llama, alpaca y ovejas, las mujeres cantan las canciones dedicadas a cada especie de animal y los varones acompañan con instrumentos musicales de cuerda, tales como la guitarra, mandolina, etc. (ver ejemplo $\mathrm{N}^{\circ} 2$ ).

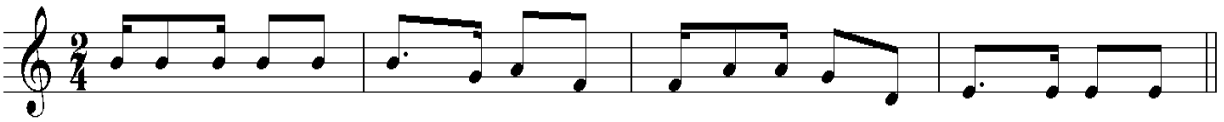

Ej. N ${ }^{\circ}$ 2. Canto a la ganadería andina (por mujeres aymaras). Recopilación y transcripción de Manuel Mamani. El texto dice: "Jichur, mayurway, tatala, juman urumaway, talata", y su traducción es: "Este lapso de tiempo, vida, serán tus días, mi vidita”.

\section{c) Carnaval andino}

Cada pueblo del área andina del norte, en los valles, en la precordillera, en las quebradas y en el altiplano, establece la presencia de la música en sus fiestas de carnaval, fiestas en las que la música es imprescindible, así como lo es en otras actividades tradicionales. Los guitarreros $\mathrm{u}$ orquestas entonan la estrofa del canto de carnaval y los asistentes, en baile de rueda, repiten en coro la estrofa. Allí nuevamente la voz aguda de las mujeres se impone sobre la voz de los hombres ${ }^{20}$.

${ }^{20}$ Experiencia personal. Cada vez que se iniciaba el carnaval, mi abuelita cantaba este canto de Carnaval: Canto de Carnaval.

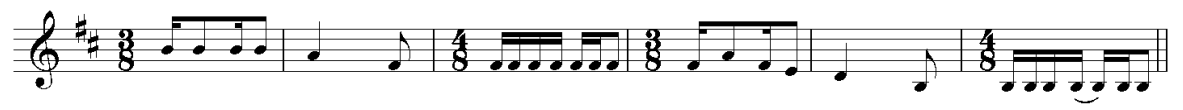

Cuyo texto dice: "Carvalaw pujix, iyawayanitay wijway sakiñani, iyawayanitay", que en español significa "Ya viene el Carnaval, ay sí, sí. Gritemos el viva”. Recopilación y transcripción de Manuel Mamani, recogido de Isabel Álvarez Pacci. 
d) Procesión de santos patronos

En las procesiones religiosas, en el desarrollo de fiestas patronales, la multitud de la población canta las canciones religiosas durante el recorrido por las calles del pueblo. La voz aguda de mujeres aymara se destaca también nítidamente en este proceso.

e) Tareas textiles, sawuña

En las tareas de urdiembre y elaboración de telares tradicionales y en la ejecución de tejidos, la mujer, mientras manipula el telar, canta canciones andinas, siempre que se encuentre sola o con acompañamiento de niñas o niños.

\section{f) Tareas pastoriles, awatiña}

En las tareas habituales de pastoreo de animales domésticos, la mujer, la niña o la joven aymara cantan las canciones de su preferencia, siempre que se encuentren solas o junto a niños o niñas en el campo pastoril.

\section{g) Labores maternales}

Generalmente, la madre joven o adulta suele cantar a sus bebés las canciones de cuna: wawar kirkiña, siempre que se encuentre a solas con su bebé. Además, entona canciones navideñas en la navidad andina (ver ejemplo $\mathrm{N}^{\circ} 3$ ).

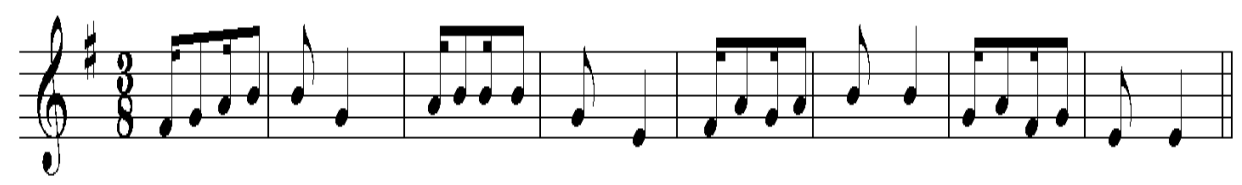

Ej. N 3. Canción navideña. Recopilación y transcripción de Manuel Mamani.

Su texto aymara es: "Wawa manulito jakill jachamnti, mä pastill churama, mä pastill churama", cuya traducción es "Niño manuelito, no llores, no llores, te daré pastilla, te daré pastilla".

h) Cofradías religiosas

En la mayor parte de los bailes religiosos que acuden a las fiestas religiosas de Las Peñas, La Tirana, y otras festividades similares, integrados por mujeres y niñas, estas últimas cantan en las plegarias musicales. Las mujeres se caracterizan sobre el grupo de danzantes con sus voces agudas.

\section{CONCLUSIÓN}

La relación de la mujer con la música es a través de cantos en los diferentes ámbitos del quehacer social, cultural y religioso. La mujer aymara no sólo vive compartiendo hábitos, ideas, creencias, sino que ella participa -en forma efectiva- en las tareas musicales, con su contraparte y con la comunidad en relación a las expectativas personales y comunales. Surge así la relación y búsqueda de equilibrio que configuran las acciones dentro del sistema de complementariedad. Así, esta 
interacción se relaciona en forma permanente y solidaria sobre la base del principio de equidad y dualidad, que sustenta el accionar e identidad de la mujer aymara.

Con este trabajo concluimos preliminarmente un esbozo del aporte que hace la mujer aymara al desarrollo musical. Esperamos abrir debate en este accionar femenino y continuar con nuevas investigaciones, con el propósito de colaborar en el desarrollo de la música andina. Este ensayo contribuirá en el quehacer musical de acuerdo a las normativas sociales y culturales andinas, dentro del campo antropológico musical.

\section{BIBLIOGRAFÍA}

ARnold, DENISE

1994 "Hacer al hombre a imagen de ella: Aspectos de género en los textiles de Qaqachaka”, Chungará, XXVI/1, pp. 79-115.

EsPinOZA S., WALDEMAR

1990 Los incas: Economía, sociedad y estado en la era del Tawantinsuyo. Lima: Amaru-Editores.

GaVILÁN, VIVIAN

1996 Mujeres y hombres en Isluga y Cariquima: Una aproximación a las relaciones de género entre los aymaras/norte de Chile. Quito: FLACSO.

Harris, Olivia

1978 "Complementary and Conflict", Sex and Age as Principles of Social Differentiation. J. La Fontaine (ed.). Londres: ASA 17, pp. 21-40.

1980 "The Power of Signs, Gender, Culture and the Wild in the Bolivian Andes", Nature, Culture and Gender. Carol MacCormack/Marilyn Strathern (eds.). Cambridge: Cambridge University Press, pp. 70-94.

ISBELL, JEAN BILLIE

1987 "La otra mitad esencial: Un estudio de complementariedad sexual andina", Estudios andinos, XXXI/12, pp. 37-56.

Mamani, Manuel

1989 Structure of the Livestock Parking Ritual in the Chilean Andes. Tesis de maestría. Gainesville: Universidad de Florida, EE.UU.

1999 "Chacha-Warmi: Paradigma e identidad matrimonial aymara en la provincia de Parinacota", Chungará, Vol. XXXI/12, pp. 307-317.

Platt, Tristan

1988 "Pensamiento político aymara", en Raíces América: El mundo aymara. Xavier Albó (ed.). Madrid: Alianza Editorial/UNESCO, pp. 365-450. 